\title{
L'art dentaire chez les Étrusques
}

\section{RÉSUMÉ}

Stéphane MONIER

Thibault MONIER

Membre titulaire de l'Académie nationale de chirurgie dentaire,

Chargé de cours à la faculté de chirurgie dentaire Paris Descartes.

Avec la collaboration du :

Pr. Danielle GOUREVITCH

Directeur d'études

à l'École Pratique des Hautes Études
Les auteurs procèdent à une présentation de la pathologie et de la thérapeutique dentaire chez les Étrusques en effectuant une lecture critique de l'ouvrage de Gaspare Baggieri et de Marina di Giacomo, publié à Rome en 2005, Odontoiatria dell'antichità in reperti osteodentari e archeologici (L'art dentaire antique d'après des découvertes ostéo-dentaires et archéologiques). En particulier, les descriptions des pièces prothétiques trouvées lors des fouilles archéologiques sont parfaitement illustrées par l'admirable iconographie de cet ouvrage. 


\section{Introduction}

$>$

En septembre 1993, nous avions déjà

publié un article sur l'art dentaire chez

les Étrusques dans le numéro 183 des A.O.S. Quand Hubert Ouvrard nous a demandé de faire une mise à jour des connaissances concernant ce sujet, nous avons associé à ce travail Danielle Gourevitch, Directeur d'Études à I'École Pratique des Hautes Études (Histoire de la Médecine). En effet, lors du XVIII congrès de la Société française d'histoire de l'art dentaire en 2007 à Castéra-Verduzan, elle a procédé à la lecture critique d'une partie de l'ouvrage de Gaspare Baggieri et de Marina di Giacomo publié à Rome en 2005, Odontoiatria del I'antichità in reperti osteo-dentari e archeologici
(L'art dentaire antique d'après des découvertes ostéo-dentaires et archéologiques). II s'agit de la compilation d'une suite d'articles qui n'ont pas été révisés pour faire un ensemble et qui couvrent une assez vaste période sans unité. Le chapitre sur la prothèse dentaire chez les Étrusques est particulièrement intéressant et présente une iconographie nouvelle, des pièces originales que nous présenterons ici.

Gaspare Baggieri est professeur d'histoire de la médecine dans le Service de recherche anthropologique et paléontologique du Ministère de la Culture, à Rome.

Marina di Giacomo, docteur en lettres et en histoire, travaille avec G. Baggieri

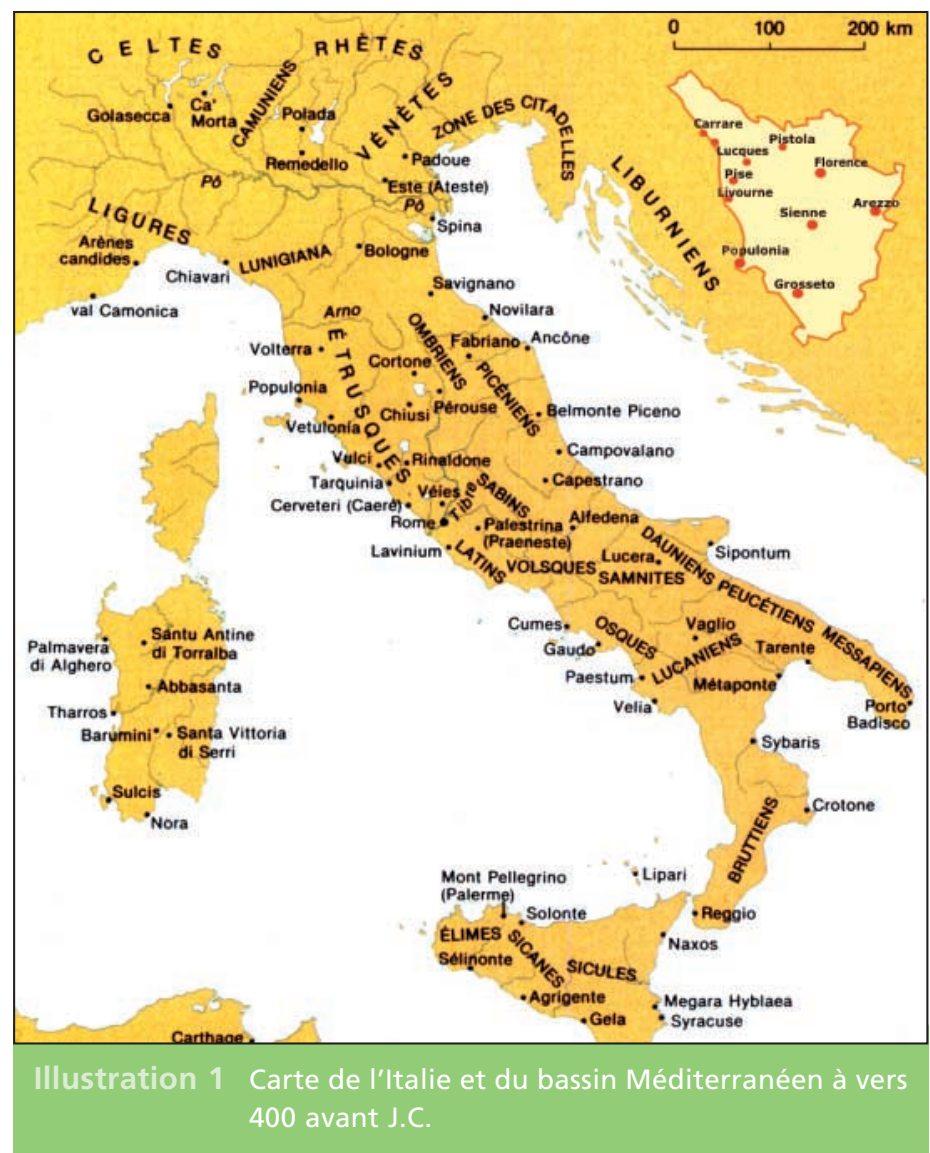




\section{L'origine des Étrusques}

Venus, selon Hérodote, d'Asie Mineure (de Lydie), pour s'installer en Toscane au VIIII siècle avant J.C., fervents adeptes des modes grecques, les peuples étrusques fondèrent Rome, en furent les éducateurs mais durent, par la suite, se soumettre à sa loi.

La civilisation étrusque apparaît définitivement constituée au début du VIIe siècle avant notre ère. Comme tous les pays du bassin méditérranéen, la péninsule italique vit s'installer de nombreuses colonies grecques et carthaginoises. Les Étrusques se trouvèrent donc en présence d'influences multiples, parfois prédominantes, mais jamais exclusives.

\section{La société étrusque}

Les grandes cités étrusques, au nombre de douze, constituent la dodécapole. Elles présentent comme seul trait d'union le fond religieux et l'unité linguistique.

La structure sociale apparaît comme un système aristocratique conservateur avec les maîtres et les esclaves. Chaque cité était politiquement indépendante et gouvernée par un Lucumon qui se présentait précédé par des Licteurs portant les faisceaux de verges entourant une hache double, insignes de la royauté.

La religion est une religion révélée, possèdant ses livres sacrés et basée sur des rites. Les dieux sont compris comme des forces collectives médiatisées par les prêtres, les Haruspices qui possèdent le don de divination.

L'apogée des Étrusques se situa vers 500 av. J.C. On retrouva les traces de leur richesse dans les tumuli de Tarquinia, Volterra, Cerveteri, ornés
L'épanouissement de cette brillante civilisation correspond à l'établissement d'une thalassocratie : les navires étrusques contrôlent la mer Tyrrhénienne. Cette expansion maritime s'accompagne d'une expansion terrestre qui s'arrête au Ve siècle av. J.C. Suit alors un déclin rapide, consécutif à la montée en puissance de Rome qui soumet l'une après l'autre les cités étrusques. Les forces aristocratiques survivantes prirent le chemin du Sénat de Rome, mais l'individualité du peuple et les traditions artistiques et religieuses se perpétuèrent tout au long de l'Empire.

de fresques riches en couleur évoquant les meilleurs moments de la vie terrestre comme la chasse, la pêche et les banquets. Les Étrusques adoraient les banquets somptueux et les représentèrent fréquemment sur les murs de leurs hypogées. Lors de ces banquets, les femmes n'avaient pas qu'un rôle figuratif, mais comme l'écrit THEOPOMPE dans son Histoire : elles se mettent à table non auprès de leur propre mari, mais auprès des premiers venus des assistants et même elles portent la santé de qui elles veulent. Elles sont du reste fort heureuses et fort belles à voir... Diodore De Sicile précisait que les Étrusques ...se font dresser deux fois le jour des tables somptueuses avec tout ce qui contribue à une vie délicate... Toutefois, le luxe déployé lors de ces banquets représentés dans les tombeaux et qui avait quelque chose de scandaleux pour les 


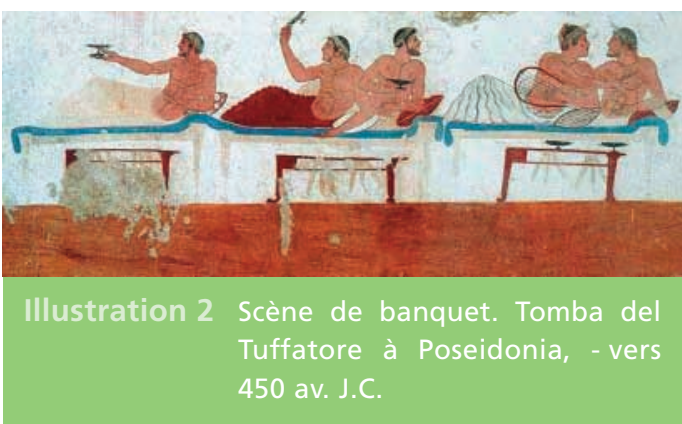

Romains plus austères, ne devait être l'apanage que des nobles ou des riches commerçants et propriétaires terriens.
Les Étrusques croyaient en une survie après la mort : incinération et inhumation des morts ont coexisté de tout temps. La survie de l'âme n'est pas liée à la conservation du corps et la sépulture assure la paix de cette âme. Les tombes regroupées en de vastes nécropoles atteignirent des dimensions gigantesques à l'architecture copiant celle des maisons des vivants. Leur découverte livra des crânes en parfait état, assurant l'étude de la pathologie dentaire dont ils pouvaient être affectés.

\section{La pathologie dentaire}

\section{L’abrasion}

Comme dans la majorité des populations antiques, les crânes étrusques montrent une abrasion importante des tables occlusales, généralisée à toute la denture, même chez les sujets jeunes. Les dents les plus abrasées sont les premières molaires maxillaires et mandibulaires du fait de leur éruption précoce et de leur rôle essentiel dans le blocage de l'occlusion en denture mixte. Les dents les moins usées sont, quand elles sont présentes, les dents de sagesse. Cette usure confère à l'arcade un aspect hélicoïdal, les surfaces occlusales prenant la forme d'une hélice dont le pas se situe au niveau de la seconde molaire maxillaire ou mandibulaire. Il en résulte que la face occlusale des dents situées avant la seconde molaire maxillaire est orientée du côté lingual; les secondes et troisièmes molaires, elles, l'ayant

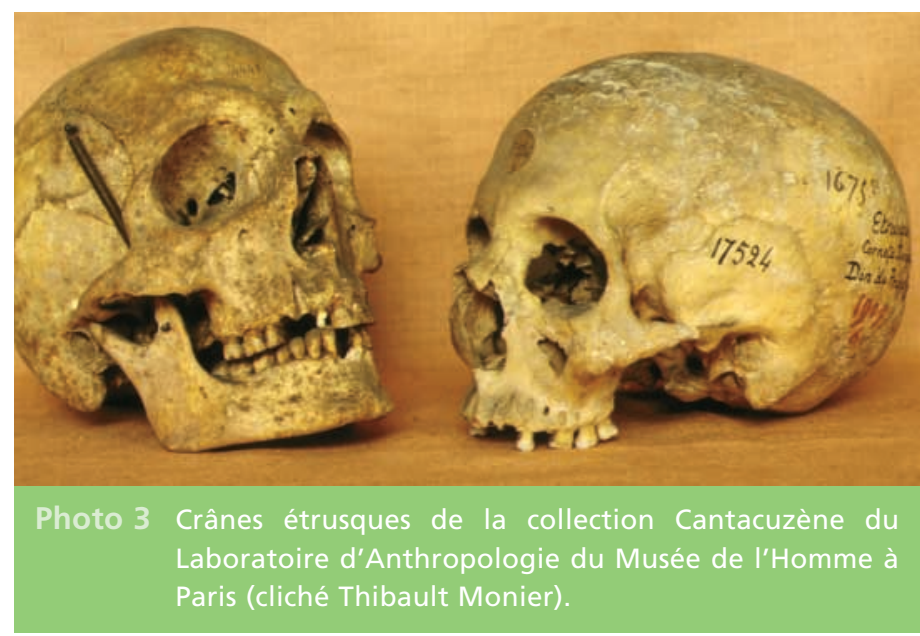




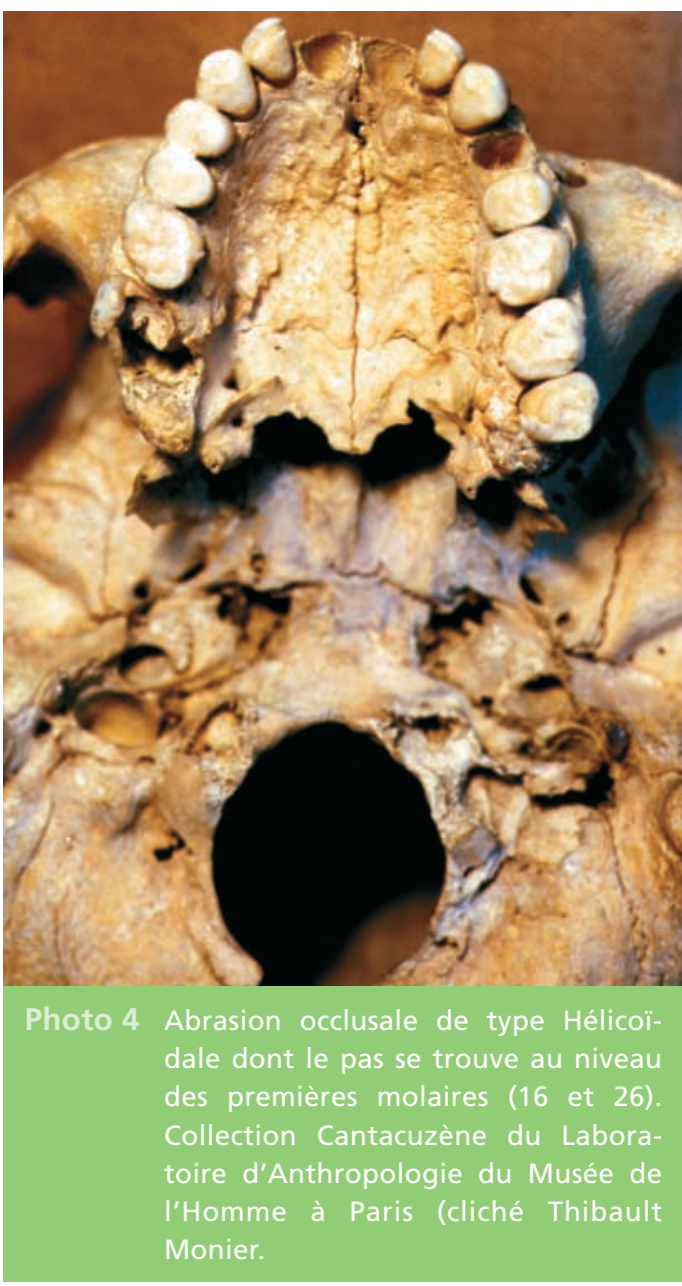

orientée du côté vestibulaire. Cette usure concerne également les faces proximales, ce qui entraîne avec l'âge un raccourcissement progressif antéro-postérieur de l'arcade.

L'usure occlusale n'étant que partiellement balancée par la formation de dentine réactionnelle au niveau du plafond des chambres pulpaires, il y avait rapidement effraction puis nécrose des dents, compliquée souvent de lésions périapicales. Les surfaces occlusales très abrasées présentent trois types de reliefs : horizontal, concave ou oblique. C'est dans le second cas, également appelé «en cupule» que l'effraction pulpaire est la plus souvent rencontrée.
L'origine de cette abrasion ne doit pas être recherchée sur le plan physiologique, mais plutôt sur le plan alimentaire, en particulier du fait de la présence de nombreuses particules minérales dans le pain.

Pline, lors d'un séjour à Carthage, a écrit que les autochtones écrasaient les grains de blé avec un pilon et y ajoutaient de la brique, de la craie ou du sable avant de les moudre. On peut donc penser que tous les pays du Bassin méditerranéen agissaient de même.

Les Étrusques appréciaient les bouillies de toutes sortes, et les recettes qu' Apicius nous à laissées concernent des viandes bouillies. L'alimentation était donc essentiellement molle, ce qui, outre le rôle joué dans l'étiologie des parodontopathies, est un facteur favorisant I'usure occlusale. En effet, les aliments durs interdisent aux dents antagonistes de se rencontrer, alors que les aliments mous favorisent cette rencontre lors de la mastication, les dents s'usant alors les unes contre les autres. Les échanges commerciaux entre les pays du bassin méditérranéen qui se développent dés le VIII siècle av. J.C. entrainent une modification du régime alimentaire : le pain, le miel, les fruits secs font leur apparition sur les tables étrusques et leur consommation entraine le développement des caries.

\section{Les caries}

Le sucre en tant que tel n'était pas connu, mais les Étrusques faisaient un emploi fréquent du miel d'abeille ou de ce qu'ils appelaient le «miel de datte» pour sucrer différents plats et même le vin. Les collections ostéologiques étrusques en Italie, étudiées par Baggieri, présentent plus de 1300 pièces dont environ 600 proviennent de la nécropole de Pontecagnano en Campanie. Il a constaté que le taux de carie augmentait sensiblement entre le IVe (3 à $4 \%$ ) 
et le 1 er siècle ( 5 à $6 \%$ ) avant notre ère, certainemant du fait d'un enrichissement du régime alimentaire.

Les dents antérieures sont peu cariées car les Étrusques ne consommaient pas de fruits acides et sucrés comme les oranges et les citrons qui provoquent une atteinte du groupe incisivo-canin maxillaire et les dents peu ou pas abrasées sont les seules à être cariées car l'abrasion entraînant la disparition du relief occlusal supprime les sites de rétention de la plaque dentaire.

\section{Les complications}

\section{osseuses}

Après avoir éliminé les dents perdues postmortem, aux alvéoles parfaitement intactes, nous avons observé des maxillaires présentant des espaces édentés où l'os était parfaitement cicatrisé. Leur absence peut résulter des complications des lésions précédemment décrites :

- l'abrasion provoquant une nécrose pulpaire, des lésions périapicales avec destruction de I'os parodontal (cas le plus fréquent) ;

- les caries, compliquées d'une atteinte pulpaire et périapicales ;

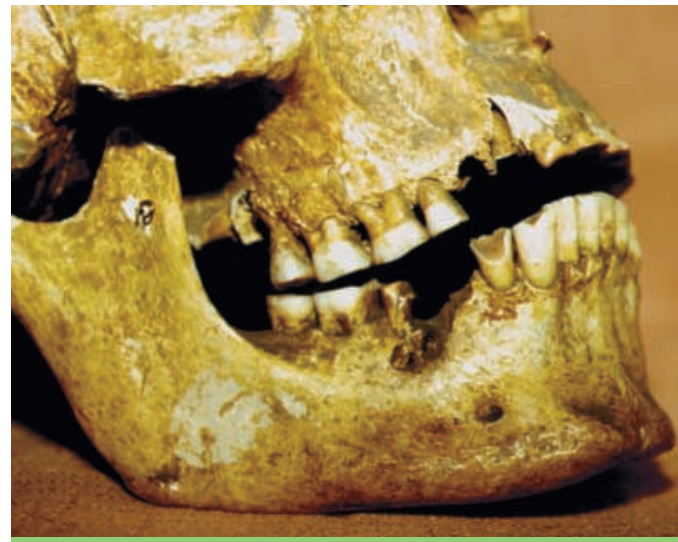

Photo 5 Complication osseuse, de l'évolution d'une lésion carieuse. Cette intéressante photo montre l'évolution d'une lésion carieuse au niveau de 46, avec développement d'un foyer périapical et destruction de la corticale externe vestibulaire des racines mésiales perdues. Notez l'abrasion importante des tables occlusales et l'alvéolyse horizontale généralisée. Collection Cantacuzène du Laboratoire d'Anthropologie du Musée de I'Homme à Paris (cliché Thibault Monier).

- les parodontopathies, avec dénudations radiculaires partielles puis totales.

Quant à savoir si certaines dents mobiles furent extraites, rien ne nous permet de l'affirmer du fait de l'aspect identique de l'os cicatrisé après perte naturelle d'une dent ou intervention humaine.

\section{La thérapeutique dentaire}

Nous ne possédons que très peu de documents concernant les pratiques médicales en Étrurie.

Les seuls renseignements qui nous soient parvenus sont quelques phrases contenues dans les textes d'auteurs latins, mais postérieurs à la période d'indépendance. Ainsi Théopraste (370-380 av. J.C.) écrivit dans son Histoire des Plantes :
Aeschyle établit dans les Élégies que l'Étrurie est riche en médecins et que la race étrusque est une de celles qui cultivent particulièrement la médecine.

Les Haruspices jouaient un grand rôle dans la pratique médicale: médecine et religion étaient étroitement liées et la magie tenait une place prépondérante pour écarter les 
mauvais sorts. La maladie devait être considérée comme un dérangement de l'ordre universel et les livres rituels de "l'Etrusca Disciplina» renfermaient des rites destinés à soulager les malades.

\section{Les bouches votives}

D'après l'Etrusca Disciplina, série d'ouvrages de référence pour les membres du clergé, la vie humaine atteignait au maximun 84 ans divisés en douze fois sept ans. Tant que l'être humain n'avait pas atteint dix fois sept ans, il pouvait conjurer le destin par des rites propitiatoires. Les fouilles pratiquées dans les temples ont mis à jour des fosses votives contenant des millions d'ex-voto, en bois, bronze, pierre ou le plus souvent en terre cuite représentant un organe ou une partie du corps.

Cet ex-voto en terre cuite figurant une bouche ovalaire dégageant largement les deux arcades laisserait penser que, comme le reste des pratiques médicales, la thérapeutique dentaire consistait essentiellement en des incantations magiques.

Ces représentations figurant un élément du corps humain malade étaient déposées dans

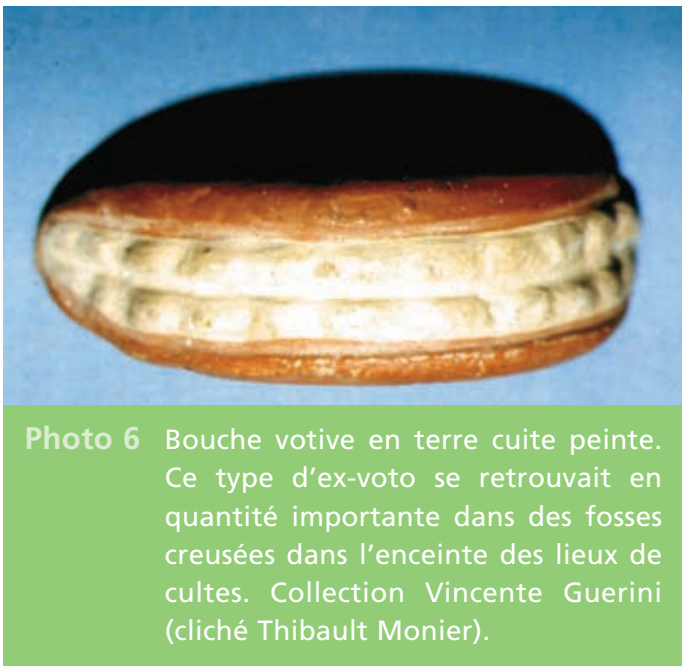

les temples afin d'obtenir une guérison, peutêtre par transfert du mal du patient vers l'exvoto, ou pour remercier un dieu d'une guérison censée être divine. Réalisés en terre cuite, moulés, fabriqués en série, ils pouvaient presque indéfiniment être reproduits, ne coûtaient certainement pas cher, et étaient extrêmement répandus : quand Danielle Gourevitch était membre de l'Ecole Française de Rome elle a constaté qu'ils étaient stockés «comme des tas de charbon» dans les sous-sols du musée des thermes! On pouvait ajouter des détails au couteau, si besoin était dans l'argile encore molle, et personnaliser l'ex-voto selon les désirs de l'acheteur. Aujourd'hui leur datation est difficile car à partir d'un moule d'origine on peut répéter indéfiniment les épreuves au cours des siècles.

Si des daviers, curettes et élévateurs furent mis à jour dans les ruines de Pompéï ensevelies sous la lave en 79 après J.C., les métropoles étrusques en furent plus avares. Les fouilles d'un tumulus inviolé ont mis à jour une pince dont selon Corti l'usage ne peut être que dentaire. Toute civilisation a possédé ou possède encore ses "arracheurs de dents» et il est probable que les Étrusques furent à même de sou-

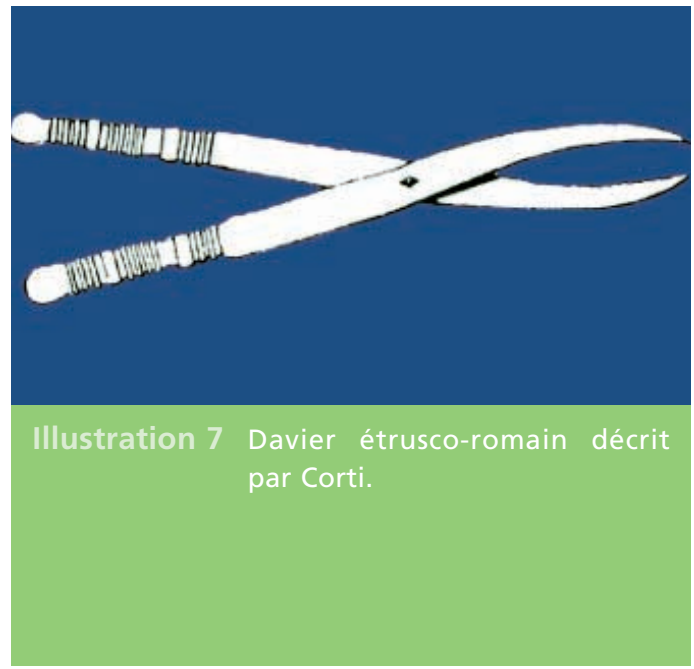

Actualités Odonto-Stomatologiques - $n^{\circ} 243$ - septembre 2008 
lager leur contemporains en extrayant les dents douloureuses, cet acte chirurgical ne nécessitant que peu de matériel et de connaissances scientifiques.

\section{La métallurgie}

Les ressources de l'Étrurie en minerais furent à l'origine de sa puissance. Les tombes de l'époque villanovienne contenaient déjà de nombreux objets en bronze, alors que les tombes grecques de la même époque n'en renfermaient pas encore. Les gisements de fer de l'île d'Elbe furent exploités surtout entre le VIIe et le Ve siècle av. J.C. Ce fer était utilisé pour la fabrication d'objets manufacturés, dans des haut fourneaux qui amenèrent certains archéologues à considérer la ville de Populonia comme la «Pittsburg de l'Antiquité». Le cuivre, le plomb argentifère et l'étain étaient également travaillés, de même que l'or. Les tombes du VIIe et du VIe siècle livrèrent de nombreux bijoux tels bagues, boucles d'oreille, pectoraux et pendentifs témoins de la maitrise des techniques du martelage, du poinçonnage et surtout du filigrane et de la granulation.

La technique de la granulation consiste à semer sur la surface à décorer des milliers de granules d'or minuscules et les y fixer par une soudure qui n'en altérait pas la finesse. Ces petites granules atteignent jusqu'à deux dixièmes de millimètre.

Cette maîtrise des orfèvres étrusques les conduisit à mettre leurs compétences au service de l'art dentaire.

\section{Les prothèses dentaires}

On a déjà beaucoup écrit sur les prothèses dentaires étrusques et le débat est très souvent passionnel, sur le point de savoir en particulier si elles sont fonctionnelles ou purement esthé- tiques: elles disparaissent après la période étrusque, ce qui est surprenant si elles devaient flatter d'autres nécessités que la vanité !

Des répliques de prothèse étrusques ont été réalisées au début du $X X{ }^{e}$ siècle par le Dr Vicente Guerini de Naples d'après les pièces trouvées dans les nécropoles de Tarquinia et d'Orviéto. II les a offertes au Musée du centre Charles Godon de Paris, où nous les avions photographiées. Nous vous les présentons avec les photographies des pièces originales publiées par Gaspare Baggieri et Marina di Giacomo dans leurs diverse publications.

Deux types d'appareillages ont été retrouvés sur des maxillaires : les contentions et les ponts fixes destinés à remplacer une ou plusieurs dents absentes ou à éviter la version des dents bordant une zone édentée.

\section{$>$ Les contentions}

On constate donc que si le traitement chirurgical des parodontopathies n'existait pas, les Étrusques savaient cependant réaliser dès le $\mathrm{Vle}$ siècle avant notre ère des appareils de contention fonctionnels, ce qui nécessitait un très haut niveau d'habileté manuelle et des connaissances dans le travail de l'or.

\section{- La contention de Chiusi (Città della Pieve)}

Ce crâne et cette mandibule sont passés entre les mains d'un collectionneur privé qui finit par les remettre aux autorités en 1905. Datés des Ve-IVe siècles, ils sont aujourd'hui au Musée archéologique national de Florence. La prothèse était en place sur un crâne féminin (femme d'environ 18-25 ans) et en assez bon état de conservation.

La mandibule possède encore toutes ses dents, sauf l'incisive centrale gauche (31), mais l'absence d'espace fait penser à une possible agénésie, ou à une perte précoce avec migration 


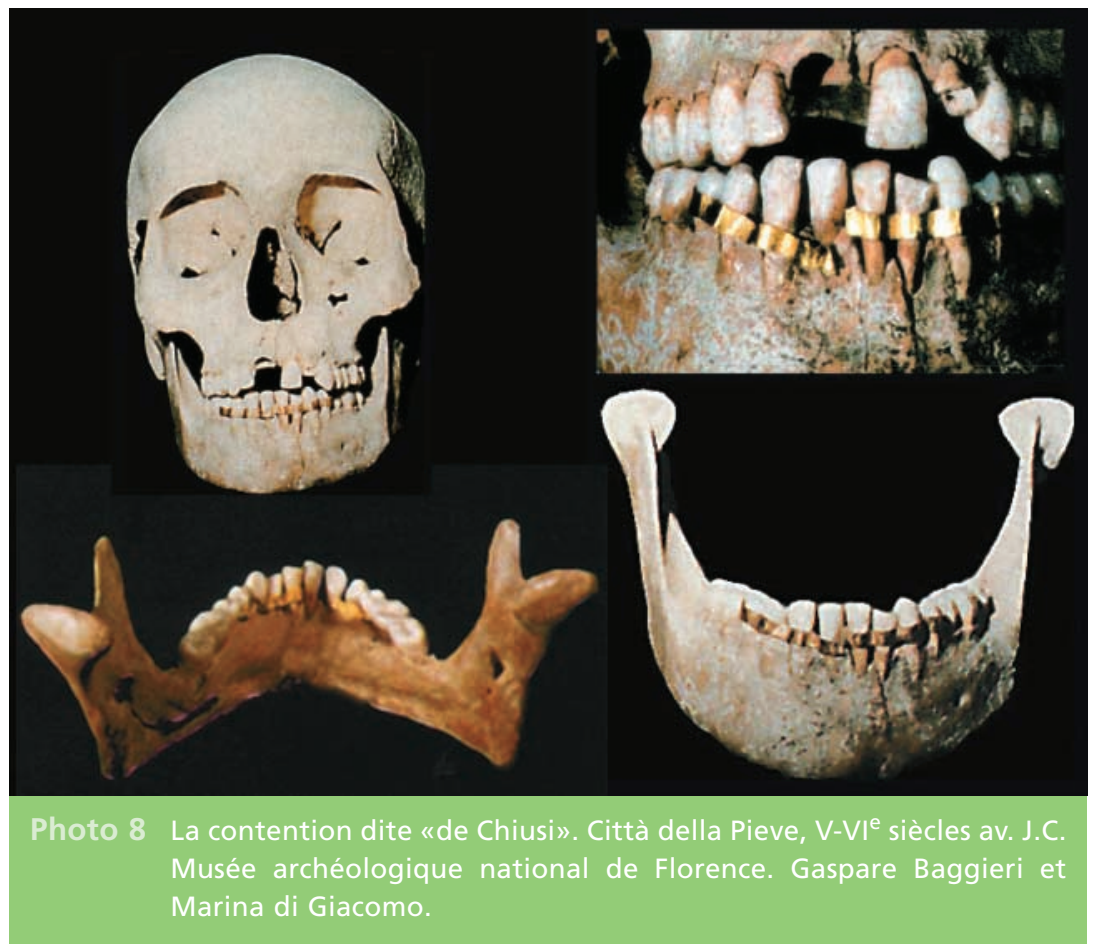

des dents adjacentes comblant l'espace résiduel. Les surfaces occlusales montrent une faible usure dentaire, en corrélation avec l'âge au décès. Sur l'arcade mandibulaire, un fin bandeau en or, large d'un peu plus de $3 \mathrm{~mm}$, forme comme une sorte de ruban allant d'une seconde prémolaire à l'autre entrant et sortant autour des espaces interproximaux des sept dents intermédiaires. On constate une résorption osseuse marquée au niveau des alvéoles de 41 , $31,32,33$ et 34 . Cet appareillage aurait donc pu être destiné à éviter la mobilité des dents alvéolysées, peut-être à la suite d'un traumatisme. La composition de l'alliage renferme $98 \%$ d'or, ce qui explique la mise en place facile des bandeaux autour des collets, le métal étant mou.

\section{- La contention de Populonia}

Le Musée archéologique national de Florence possède un second exemple de contention mandibulaire provenant de la nécropole de Populonia. Le bandeau d'or est moins large

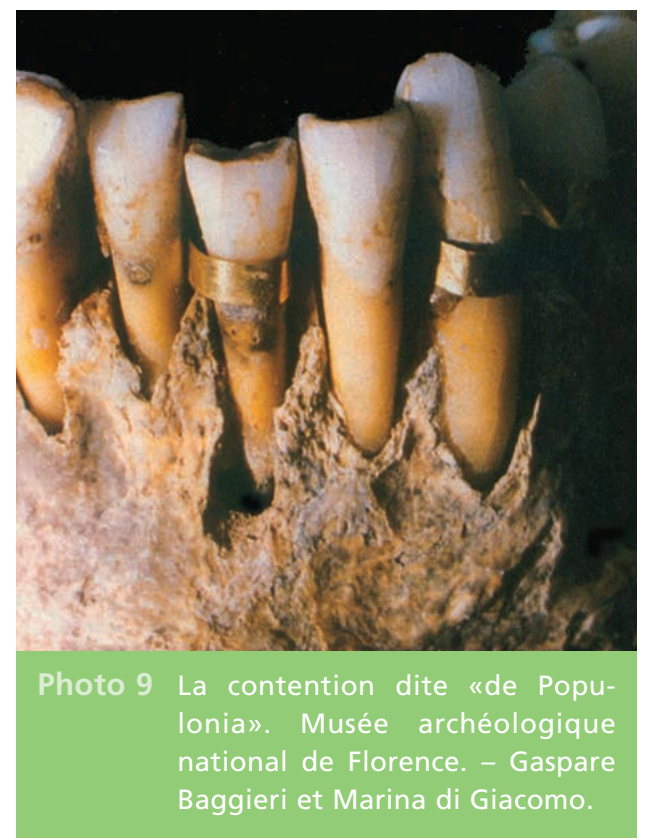

(2,2 à 2,5 mm) et la contention s'étend de la première molaire droite à la première prémolaire gauche. On observe une alvéolyse marquée du groupe incisivo-canin. La datation de la pièce et le sexe n'ont pas été précisés. 


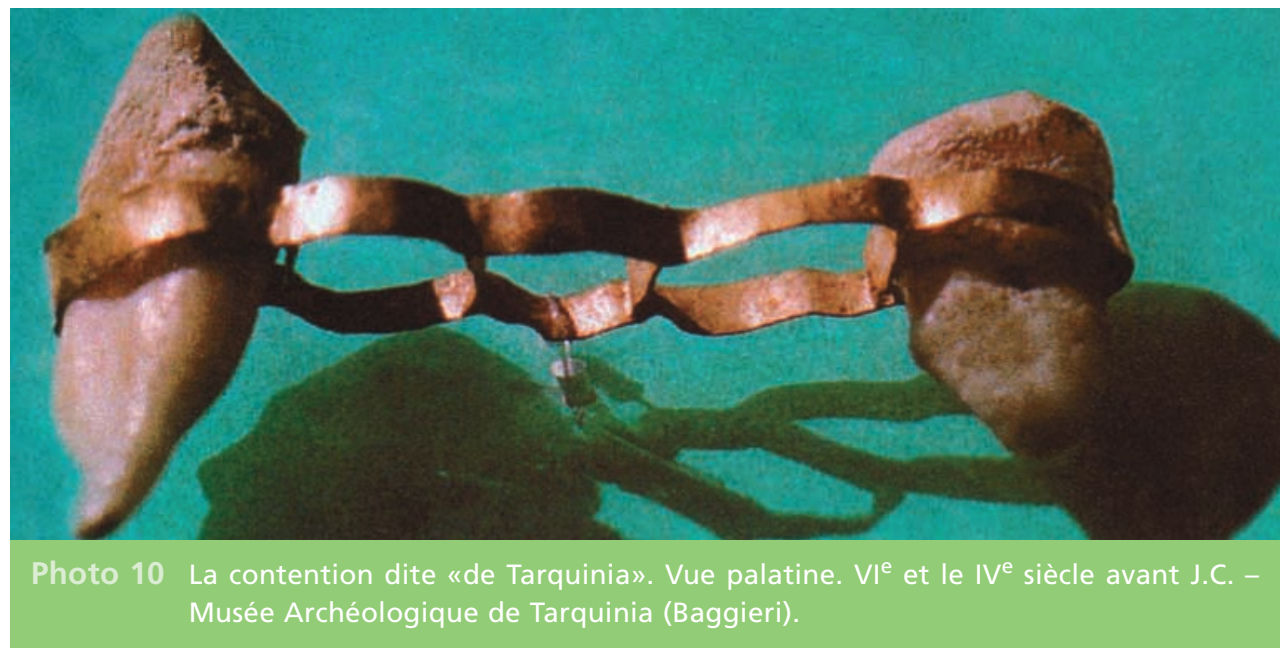

\section{- La contention de Tarquinia}

Le contexte archéologique ayant amené à la découverte de cette pièce au XVIIe siècle est mal connu. Elle est conservée au Musée Archéologique de Tarquinia.

Datée du IVe siècle avant J.C., elle est constituée d'un système de cinq anneaux en or de $3 \mathrm{~mm}$ de large qui entouraient $31,32,33,34$, et 35 .

Seules deux dents sont toujours présentes : 31 et 35. La faible usure du métal suggère que cette contention n'a pas été portée longtemps (Baggieri, 1999).

\section{- La contention d'Orvieto}

Le Musée de l'Université de Gand conserve un autre exemple de contention, sur un maxillaire supérieur, provenant de la nécropole d'Orviéto et daté du Vle siècle avant J.C.

Autour de 13, prend appui une ceinture métallique en or mou ajustée aux faces vestibulaire, distale et palatine. Elle se prolonge en mésial, présentant les bombés correspondant aux 3 incisives adjacentes et contournant la face distale de 21. Deux lamelles, orientées dans le sens vestibulo-lingual, sont

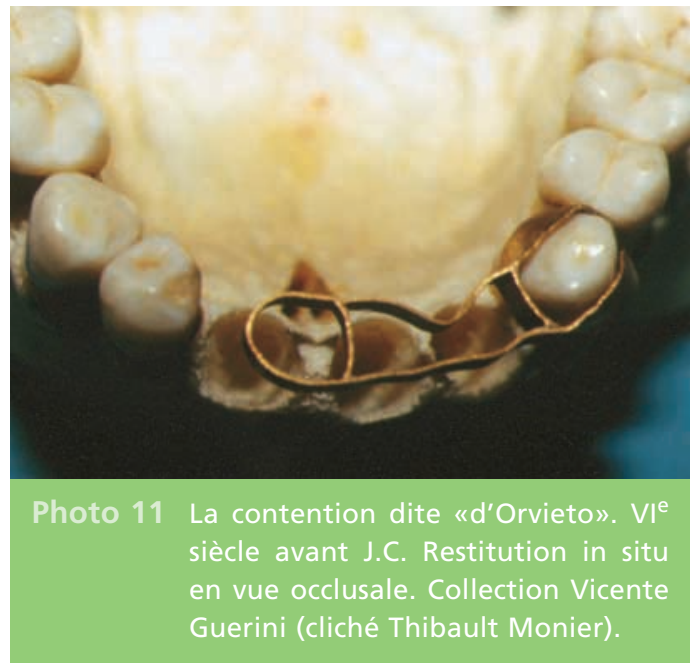

soudées à la partie interne de cette ceinture au niveau des faces mésiales de 13 et 21 . Ces deux dents parfaitement enserrées soutenaient le dispositif de contention de 12 et 11 très mobiles. Le sexe du propriètaire n'est pas déterminé.

À cette époque, les femmes étaient-elles plus «coquettes» que les hommes ? Il faudrait pour ces prothèses dites esthétiques tenter une sorte de statistiques par sexe afin de répondre à cette question ! 


\section{> Les prothèses}

Les Étrusques furent aussi capables de remplacer les dents perdues et d'éviter la migration des dents bordant les édentements.

Deux types d'appareillages furent découverts dans les sépultures étrusques :

- des mainteneurs d'espace.

- des prothèses de remplacement.

\section{- Les mainteneurs d'espace}

La première pièce, réalisée entre le Vle et le IVe siècle avant notre ère, est constituée d'un bandeau unique en or de $2,5 \mathrm{~mm}$ de large entourant 21, 22 et 23 . Son poids est de 2,5 g.
Un examen des faces externes du bandeau au microscope a permis de mettre en évidence la soudure des deux extrémités de cette bande et à révéler de nombreuses stries multidirectionnelles qui sont probablement dues à la mastication (Baggieri, 1999, Baggieri et al. 2001). Elle est conservée au Musée Archéologique de Tarquinia.

Un second exemple de mainteneur d'espace prenait appui sur l'incisive centrale mandibulaire gauche (31), la canine et la seconde prémolaire du même côté (33 et 35), évitant le comblement de l'espace correspondant à l'incisive latérale et la première prémolaire.
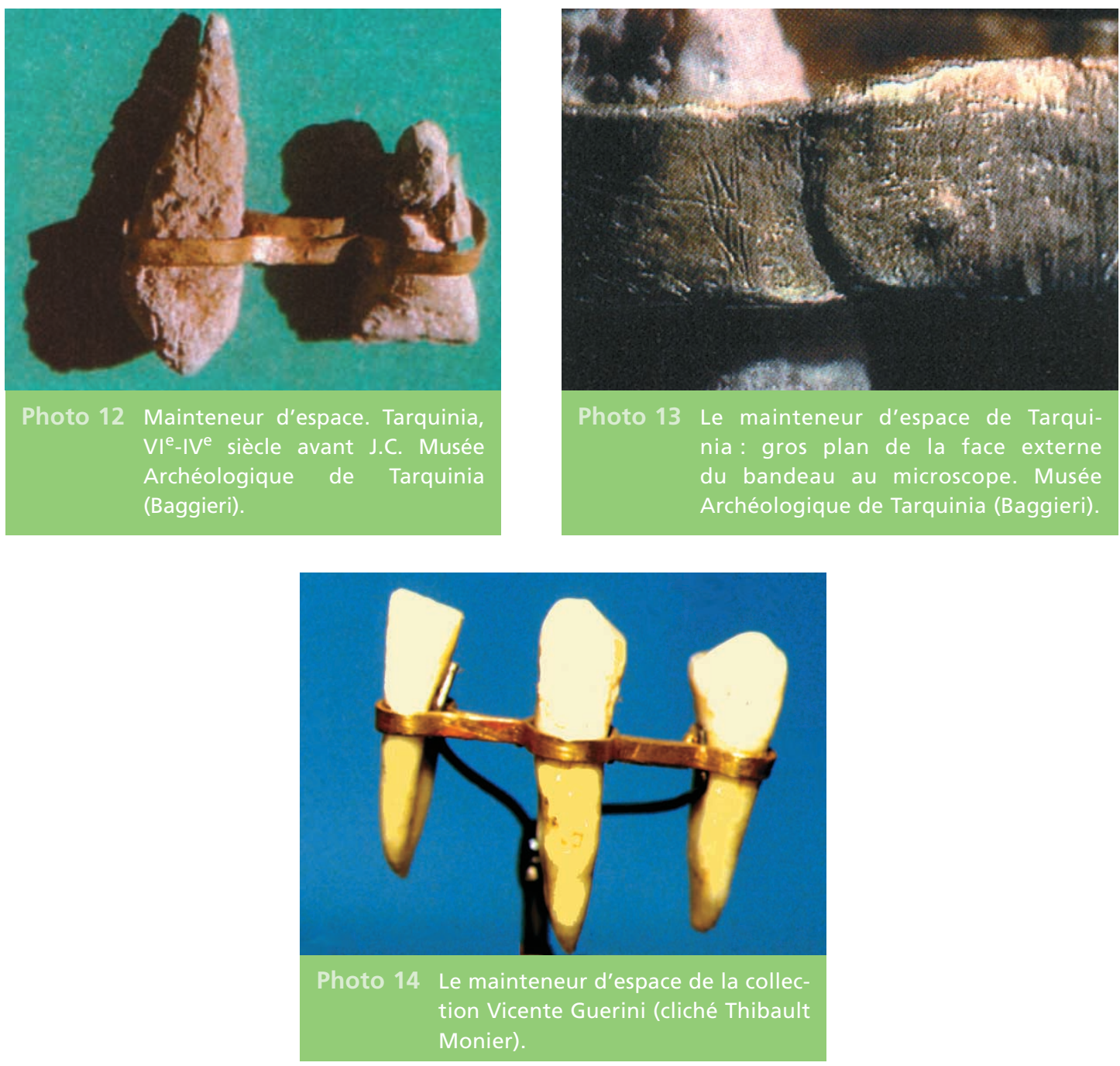
La datation et la provenance de cette pièce nous sont inconnues.

\section{- Les prothèses de remplacement}

Les «ponts» montrent quel haut degré de maîtrise des techniques prothétiques les Étrusques avaient atteint.

Formés de bandeaux ou d'anneaux d'or travaillés à la pince et soudés, ils supportaient des couronnes en or faconnées ou coulées, ou en ivoire animal et parfois humain. Elles étaient fixées à l'appareil de support par un rivet ou soudées quand elles étaient en or. L'appareil terminé, les dents étaient polies afin de ne pas léser la gencive et la rétention était principalement trouvée dans le frottement des anneaux ou des logettes d'or autour des dents supports.

\section{- La prothèse de Civitacastellana}

Elle fut découverte au XIXe siècle dans une tombe de la nécropole de Valsiarosa, une des plus riches de Falerii, de population falisque, et peut-être antérieure au monde étrusque (VIIe siècle). Malheureusement la tombe avait été saccagée et on ne dispose pas d'objets funéraires permettant une datation plus précise.

Le sujet est un homme d'environ 40 ans, dont les maxillaires ne portent plus aucune dent en place : toutes furent perdues post-mortem. Le positionnement de la prothèse pose donc un problème! Baggieri pense que la prothèse était supportée par les incisives supérieures 11, $X, 22$, et la canine gauche, la 23. En effet, il a essayé de placer l'élément prothétique en fonction des reliefs osseux des maxillaires et c'est au niveau des alvéoles de ces dents que l'adaptation était la plus probable.

Un bandeau d'or de $6 \mathrm{~mm}$ de large a été façonné de façon à épouser les faces vestibulaires et linguales de $11,21,22$ et 23 et les faces distales de 11 et 23 . Trois lamelles orientées dans le sens vestibulo-lingual ont été soudées à la face interne du bandeau principal, au niveau des espaces interdentaires créant ainsi quatre logettes.

La logette correspondant à 21 présente un rivet vestibulo-lingual destiné certainement à fixer une dent postiche. Le technicien de l'antiquité avait donc utilisé une dent, peut-être celle perdue par le patient, l'avait sectionnée au collet, puis percée dans le sens vestibulo-lingual au centre de la face vestibulaire. Une fois ajustée dans sa logette, elle avait été rivetée dans le sens vestibulo-lingual. Le poids de cette prothèse est de 1,96 g. L'alliage utilisé renfermait entre 50 et $75 \%$ d'or et 20 à $45 \%$ d'argent assurant une bonne résistance mécanique et à la corrosion.

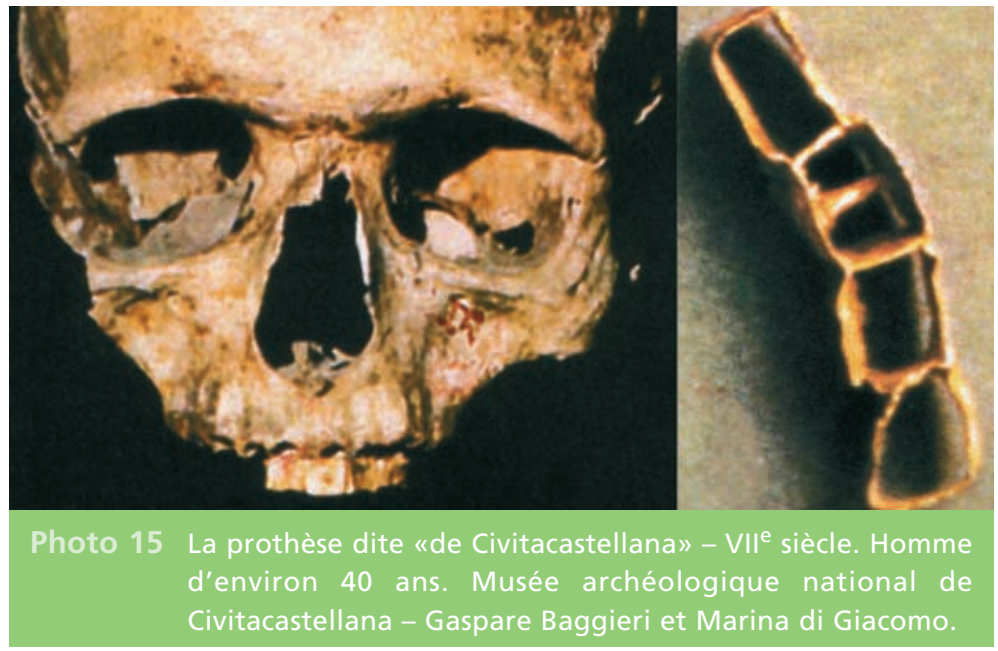




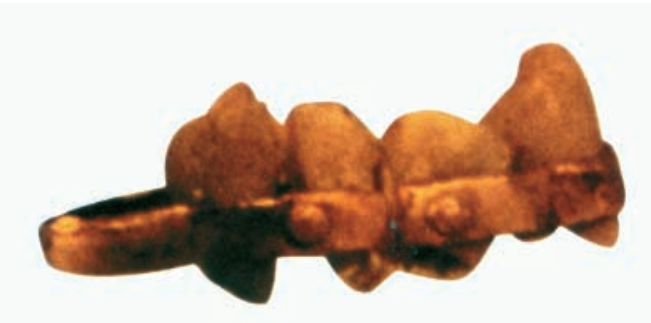

Photo 16 La prothèse dite "de Palestrina», VIle siècle. Musée Archéologique National de la Villa Giulia - Gaspare Baggieri et Marina di Giacomo.

\section{- La prothèse de Palestrina}

Privée des structures osseuses, cette prothèse proviendrait de Palestrina, près de Rome. Elle est conservée au Musée Archéologique National de la Villa Giulia.

Ce pont est constitué d'un bandeau d'environ $3 \mathrm{~mm}$ de large. II remplaçait une 21 et une 22 en prenant appui sur 11 et 23 . Les 2 dents manquantes étaient fixées dans leur logette par 2 rivets orientés dans le sens vestibulo-lingual. La 11 est manquante.

La technique de réalisation, semblable à celle utilisée dans l'exemple précédent, permettrait à Baggieri de dater ce travail également du VIIe siècle avant notre ère (Baggieri, 1999).

\section{- La prothèse de Satricum (lieu-dit Conca Le Ferriere)}

Découverte au début du siècle, dans la tombe XVIII e de la nécropole de Satricum près de Rome ce cas présente une tentative de remplacement d'une 32 perdue par une coiffe évidée en or. Elle est conservée à Rome au Musée Archéologique National de la Villa Giulia.

La dent prothétique, une capsule de $10 \mathrm{~mm}$ de hauteur est formée de 2 facettes réunies qui

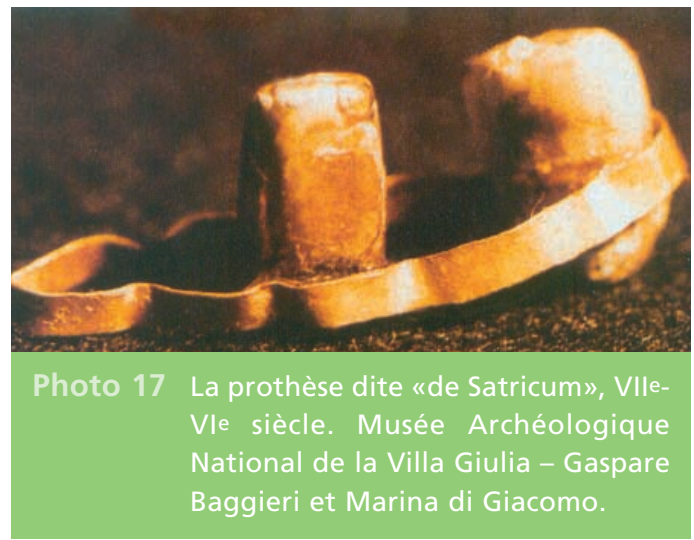

devaient recouvrir le moignon de la 32. Elle est solidarisée par des points de soudure à l'argent à un bandeau en or qui prend appui autour de 41 et 31 d'un côté, et de 33 et 34 de l'autre. Une seule dent naturelle est conservée : la 34. D'aprés Baggieri, sa face occlusale présenterait une cavité creusée afin d'y placer une obturation. La datation de ce travail serait des VIII Vle siècles. (Baggieri et al. 1993, Baggieri et al. 2001).

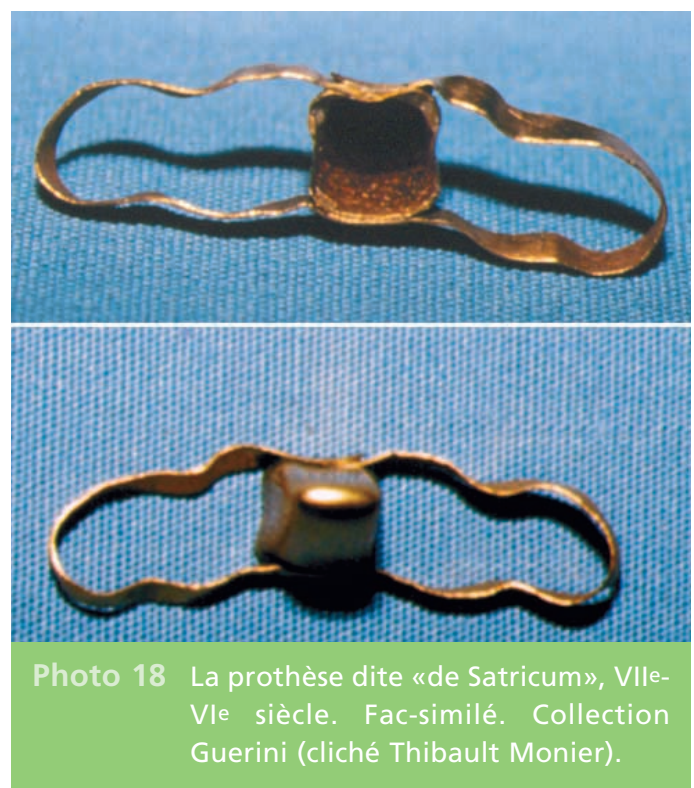




\section{Conclusion}

Théophraste et Martianus Capella nous apprennent que les Étrusques avaient acquis une grande et lointaine renommée par leur art de composer des médicaments. Dès les temps les plus reculés de l'histoire étrusque, s'instaura une liaison étroite entre pratique médicale, magie et religion. Les tombeaux de Tarquinia et de Capodimonte ont livré aux archéologues les premières prothèses dentaires fonctionnelles de I' Histoire, appareils de contention ou de remplacement en or, témoins de l'habileté des Étrusques dans le travail des métaux précieux.

L'ouvrage de Gaspare Baggieri et de Marina di Giacomo témoigne de l'essor de la paléopathologie dentaire, discipline aux progrès de laquelle Baggieri a contribué avec la découverte de l'athlète de Tarente et l'étude de sa denture (p. 73-81). Son ouvrage en italien captivera grâce à sa riche iconographie tous les praticiens souhaitant s'initier à l'étude des maladies de nos ancêtres.

Ouvrages à consulter

Baggieri Gaspare.

Appointment with an Etruscan Dentist.

Journal of the Etruscan Studies Foundation, vol. 6, pp. 33-42, 1999.

Baggieri Gaspare

et Di Giacomo Marina.

Prothèse dentaire

chez les Étrusques.

Pour la Science,

$\mathrm{N}^{\circ} 285$, juillet 2001,

pp. 60-65.

Baggieri Gaspare et Di Giacomo Marina. Odontoiatria dell'antichità in reperti osteo-dentari e archeologici.

MelAMi, Rome 2005.

ISSN 1827-8639.
Cantacuzene Georges. Contribution à la craniologie des Étrusques.

L'Anthropologie, tome XX, 1909, pp. 329-352.

Guerini Vicente. History of Dentistry. Lea \& Febinger, Philadelphia and New York, 1909.

Gourevitch Danielle. A propos d'un livre récent sur les dents et l'art dentaire dans le monde étrusco-romain. Compte rendu critique. Actes

de la société française d'histoire de l'art dentaire. XVIIe congrès.
Vol. 12, Castera-Verduzan, 2007, pp. 5 à 9.

Hus A.

Les Étrusques et leur destin. Picard, Paris, 1980.

Monier Stéphane. Médecine, pathologie, thérapeutique et prothèse dentaire chez les Étrusques.

Thèse de $2^{\mathrm{e}}$ cycle pour le Doctorat en chirurgie dentaire. Paris Descartes, 1984.

Monier Stéphane \& Thibault. L'art dentaire chez les Étrusques.

A.O.S., $\mathrm{N}^{\circ} 183$, septembre 1993. 
SUMMARY

\section{Etruscans}

\section{and the art of dentistry}

Stéphane MONIER

Thibault MONIER

Danielle GOUREVITCH

Keyvords
- critical review
- history
- pathology
- prothesis
- roman
and etruscan world

The authors review Odontoiatria dell'antichità in reperti osteo-dentari e archeologici by Gaspare Baggieri and Marina di Giacomo, and insist on some Etruscan cases of dental pathology and therapeutics. They present the most interesting examples of dental bridges according to the beautiful pictures published in this book. 


\section{Abonnement}

Pour s'abonner, composer le :

tél. + $33(0) 169181514-\mathrm{fax}+33(0) 169860678$

France et Dom-Tom (voie de surface) $\ldots \ldots \ldots . . .1$
Membre AEOS
Étudiant France (sur justificatif)
DOM-TOM (par avion)
Étranger (par avion)
(par voie de surface)
PRIX AU NUMÉRO
France
Frais de port au numéro

* Offre valable jusqu'au 31/10/08

Réglé la somme de le

Chèque bancaire / postal $\mathrm{N}^{\circ}$ ou carte $\mathrm{N}^{\circ}$

(Partie à conserver)

(Partie à joindre à tout règlement)

$\square$ Je souhaite m'abonner pour

à la Revue AOS

$\square$ Je souhaite prolonger mon abonnement pour

à la Revue AOS

MODE DE PAIEMENT :

$\square$ Paiement effectué par chèque à l'ordre de : S.I.D. - EDP Sciences - REVUE AOS

17 avenue du Hoggar - P.A. de Courtabœuf

B.P. 112 - 91944 Les Ulis Cedex A (France)

Tél. + 33 (0)169181514 - Fax + 33 (0)1 69860678

e-mail : abonnements@edpsciences.org

$\square$ Paiement par carte bancaire : $\square$ Carte Visa

Master Card

$\mathrm{N}^{\circ}$

Noter ici les 3 derniers chiffres situés au dos de la carte

Date d'expiration

Signature obligatoire

VOTRE ADRESSE :

Vous êtes: $\square$ Chirurgien-Dentiste $\square$ Stomatologue $\square$ Orthodontiste $\square$ Autre : précisez

Nom :

Prénom :

Adresse :

Code Postal

Ville

Tél

Fax

E-mail 